\title{
CONFECCIÓN TEÓRICA DE ITINERARIOS
}

\section{Autor}

\section{Resumen}

Este trabajo, quizá de forma pretenciosa, pretende sistematizar la presentación e información de itinerarios en las actividades escolares que denominamos Actividades en la naturaleza o en el medio natural.

Se aborda desde una necesaria interdisciplinariedad donde la cuestión geográfica y paisajística son materias prioritarias en el texto. Se sistematiza a través de puntos cómo: localización, acceso y retorno, duración prevista, cálculo de distancias, clima y clima locales, material necesario, flora y fauna realmente esperada, perfiles y grado de dificultad, paradas, zonas de descanso o interés, costumbres, fiestas, permisos, zonas de seguridad, actividades complementarias..

Se completa el trabajo con algunos consejos prácticos, la confección de un rutómetro y un vocabulario específico para este tipo de actividad.

Palabras clave: Itinerario, naturaleza, medio natural, marcha.

\section{Descripción y confección de itinerarios para actividades en la naturaleza}

A la hora de confeccionar un itinerario bien sea como trabajo teórico, publicación, o simplemente por curiosidad, debemos abarcar una serie de puntos de manera independiente, pero que sin embargo como conjunto nos aportará una idea bastante acertada del recorrido a realizar. Una buena presentación del itinerario propuesto, es una motivación complementaria para la realización, estancia y estudio en la zona elegida.

Los diferentes puntos que proponemos son los siguientes:

Localización, acceso y retorno. Descripción del tipo de terreno (pista, camino, sendero). Mapa de la zona.

Ejemplo: Parque Natural De Las Dunas De Liencres. ${ }^{1}$

Extensión: $194 \mathrm{Ha}$.

Situación. Sus coordenadas son, $43^{\circ} 27^{\prime} \mathrm{N}, 3^{\circ} 58^{\prime}$ W. Situado a unos $12 \mathrm{Km}$. al W de Santander.

1. CENDRERO UCIEDA A. y otros. «Guía de la Naturaleza de Cantabriar. Ediciones Estudio. Santander 1993.
Este Parque Natural declarado por decreto 101/1.986 es el primer espacio protegido de Cantabria. Situado en la margen derecha del río Pas, está constituido por la flecha litoral que en sentido $E-W$ se ha desarrollado en su desembocadura.

Al norte, el mar Cantábrico bate la abierta playa de Canallave, al sur las tranquilas aguas de la ría de Mogro ponen el contraste en una zona de gran belleza cuyo principal agente modelador ha sido el viento.

Se trata del sistema dunar mejor desarrollado del litoral Cantábrico y claramente activo en la actualidad. Las dunas de su sector oriental han sido fijadas por una repoblación de pino marítimo. Alberga una amplia red de caminos que se han dotado de mesas y bancos con objeto de atender la afluencia de visitantes que hacen de Liencres un parque claramente periurbano sometido a una fuerte presión urbana.

Las dunas presentan un perfil clásico. Frentes de avance con pendiente fuerte hacia el SE en dirección al interior, y los frentes de acumulación con pendiente suave hacia el NW de donde proceden los vientos dominantes. 
Todo el conjunto de materiales geológicos del parque está constituido por materiales arenosos cuaternarios con abundantes fósiles.

\section{Cómo llegar}

Por la autovía de Santander a Torrelavega, tomamos la salida de Boo. Transitando por esta carretera local, pronto encontraremos la desviación a la izquierda que señala Playa de Valdearenas.

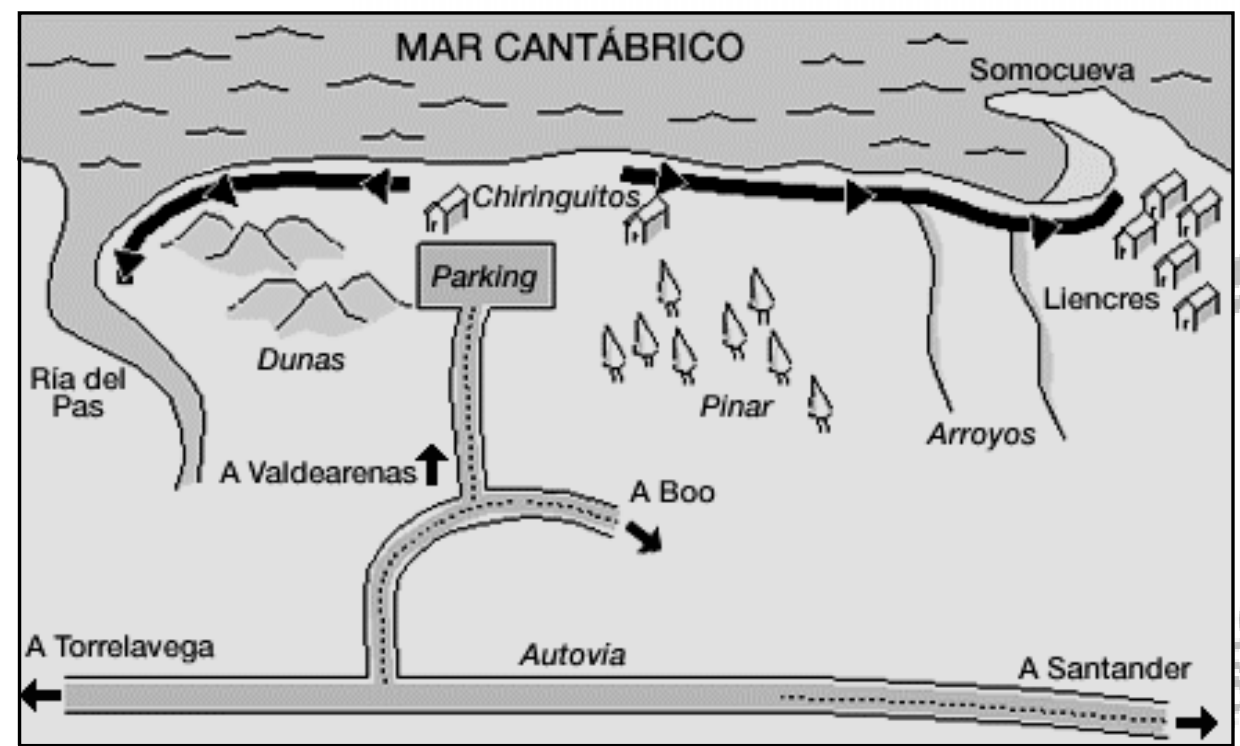

Duración y distancia a recorrer

Uno de los errores que podemos observar en la mayoría de las guías o folletos de información, es la disparidad de criterios a la hora de decidir la duración de un mismo recorrido. No es lo mismo aplicar el criterio de una marcha para montañeros avezados que de un paseo familiar festivo, por tanto, deberíamos dar unos márgenes mínimos y máximos donde probablemente estarán incluidas la mayoría de las personas que realicen el recorrido propuesto.

Mucho más simple es incluir la distancia a recorrer son varios los métodos que podemos utilizar, a partir de las escalas gráficas, el curvímetro, un hilo, o simplemente una regla, sirven para calcular los km a recorrer.

Siguiendo con el ejemplo anterior podemos decir que el recorrido es de unos diez kilómetros, ida y vuelta. La duración estimada está comprendida entre 1 y 2,30 horas.

No estaría de más recordar que la línea recta en los mapas, no siempre en las marchas, se corresponde con una menor duración de las mismas, ni de un mejor piso o trazado, no estaría de más buscar e identificar los obstáculos a evitar (pantanos, desniveles, etc.).

Época favorable para su realización y clima

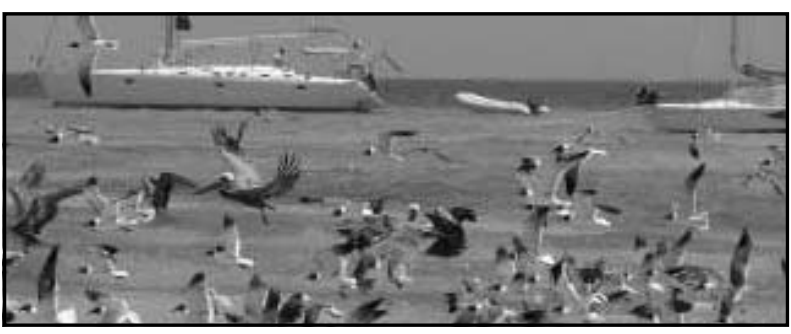

La época favorable y el clima son dos factores estrechísimamente unidos, el grado de dificultad aumentará si en invierno realizamos una ascensión, o en verano elegimos realizar una travesía en ambientes áridos.

Evidentemente no debemos olvidar además, la dureza representada por la distancia a recorrer. Por ello, a partir de la media montaña, y con la participación de personas no expertas, se deberá respetar los meses de invierno, finales del otoño y comienzo de la primavera. De igual forma los meses de verano no son recomendados para terrenos de clima continental, donde no existan láminas de agua o vegetación, donde poder aliviar las temperaturas.

No obstante, existen en España zonas donde apenas influye la condición estacional, incluso en invierno la presencia algún anticiclón, o algunos climas locales suavizan las condiciones meteorológicas para poder disfrutar de una exitosa actividad.

Lo mismo puede ocurrir si la actividad planteada es de remo o piragüismo, donde la meteorología influye si cabe, de manera más poderosa. La previsión de lluvias en la zona escogida, va a provocar crecidas en los ríos y aumentará el riesgo para las personas poco expertas, siendo sin embargo, motivo justificado para aquellas personas gustosas de aguas bravas.

Como siempre una buena previsión y un buen conocimiento de la zona, soslayar riesgos innecesarios (avenidas, aludes, oleaje, congelaciones, etc.), se hace necesario.

Siguiendo con el ejemplo que nos ocupa, la información para este punto está recogida del Observatorio Meteorológico del Cantábrico:

\section{Datos climáticos:}

Precipitación anual media $1.250 \mathrm{~mm} /$ año.

Temperaturas medias: Mes más frío: Enero 9,6 $6^{\circ} \mathrm{C}$ Mes más cálido: Agosto $19,5^{\circ} \mathrm{C}$

Ya, a simple vista podemos prever que nos puede llover pero las temperaturas no van a ser impedimento para completar con éxito esa marcha.

\section{Material necesario:}

Resulta obvio, que el material a utilizar va a depender en gran medida de la actividad a realizar. Para una marcha de corta duración, en terreno sin grandes desniveles, y buenas salidas alternativas, sólo es necesario ropa cómoda y zapatillas deportivas.

El asunto se complica a medida que complicamos también la actividad, pero no hasta el punto de cargar con excesivo material en nuestra mochila, duplicando muchas veces el uso potencial del material. No debemos confundir un buen tiempo y sol con despojarse de ropa, porque las quemaduras (aunque llevemos un protector), harán su 
aparición, y no digamos si llevamos pantalones cortos, donde además de las quemaduras los arañazos y picaduras pueden molestarnos durante bastante tiempo después de la marcha.

Es más importante unas buenas botas o un polar que la mochila o bidón último modelo.

No obstante, no debe faltar en ningúna actividad, un mapa, brújula o GPS, prismáticos, botiquín, teléfono portátil (todo ello de uso común), chubasquero o capa y alguna camiseta de repuesto. Evidentemente la comida y el agua no deben faltar en cada mochila aunque tengamos previsto pasar por fuentes, pues es mejor renovar el agua que echar en falta su ausencia.

\section{Fauna y Flora:}

No se trata en este apartado de realizar un compendio exhaustivo de biología, ni de incluir aquellas especies que pudieron existir, pero que en la actualidad no podremos ver. También debemos omitir (al menos publicar), aquellas especies endémicas y de rigurosa protección, ya que serían objeto más que probable de su desaparición.

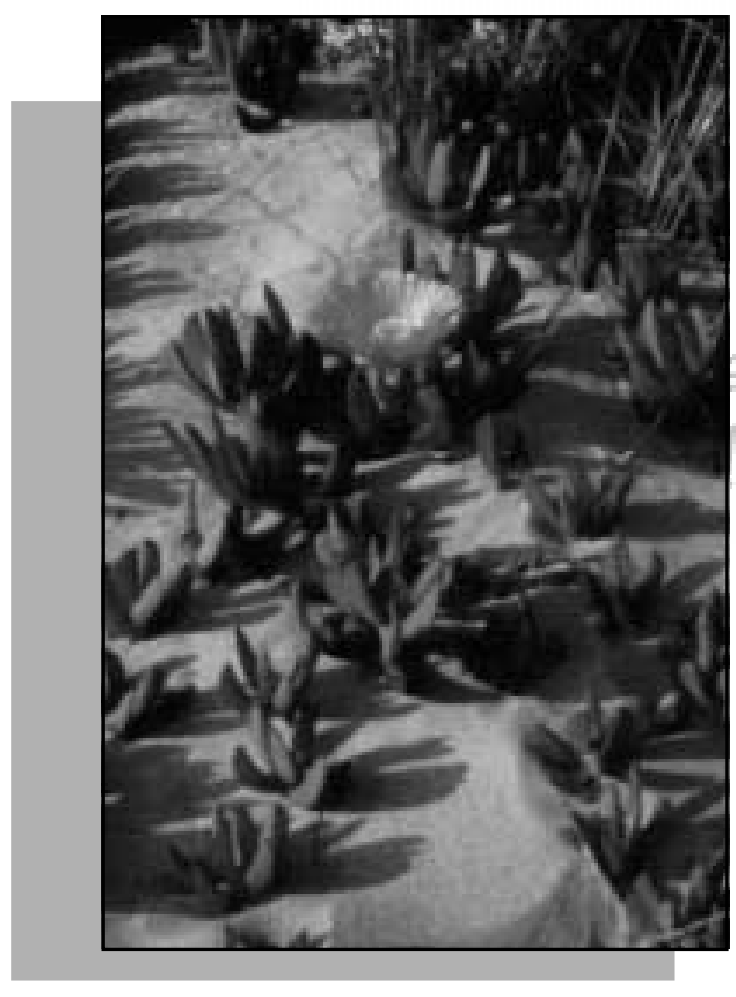

Existen numerosas guías de donde poder sacar la información necesaria, y como siempre nuestro instinto y conocimiento de la zona nos ayudarán en la elección de la fauna y flora con mayor probabilidad de encuentro.

En nuestro caso, la información que podemos adjuntar puede ser del tipo siguiente:

Ecosistemas principales. Sistema dunar, playa.

\section{Ámbito: Litoral}

La vegetación dunar está muy bien representada, variando con la orientación la distribución de las distintas especies, entre las que cabe destacar: Agropyron junceiforme (Grama del norte), Euphorbia peplis (Lechetrezna), Cakile marítima(Rucamar), Ammophila arenaría
(Barrón), Honkenya peploides (Arenaría de mar), Helichrigsum stoechas (Manzanilla bastarda).

En cuanto a la fauna, su principal valor lo constituyen las aves limícolas migratorias, principalmente el Chorlito gris y el Correlimos común. Como invernantes habituales podemos citar: Cormorán grande, Garza real, Polla de agua, Rascón y Martín Pescador. Entre las gaviotas la más abundante en Liencres son la Gaviota patiamarilla y la Gaviota sombría.

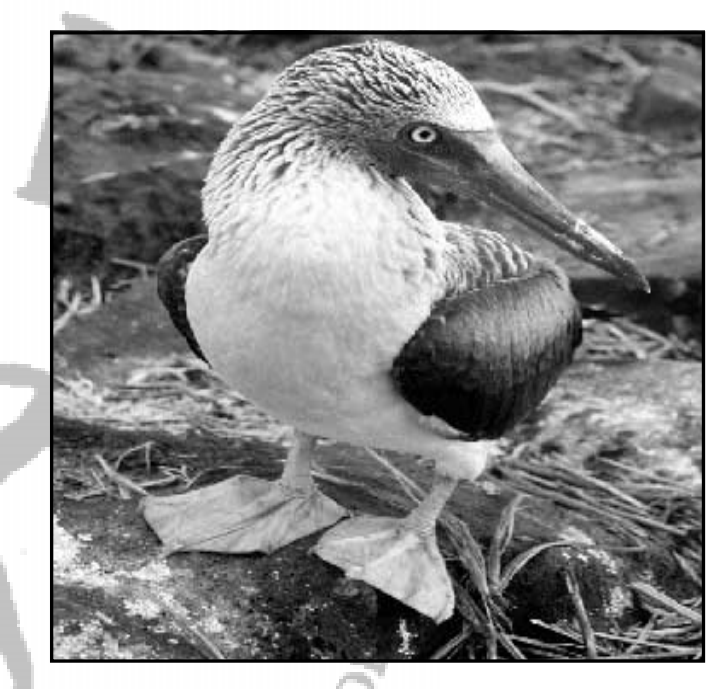

Perfiles: Grado de dificultad

Método para interpretar el perfil de una sección transversal del terreno:

¿Cómo conocer las pendientes, entre los puntos $A$ y $B$ del mapa de abajo, para planificar la marcha?

1. Poner el borde recto de un papel vegetal o poliéster o una regla sobre el mapa de manera que atraviese la zona de $A$ a B.

2. Marcar en el borde los puntos $A$ y $B$

3. Mantener fijo el papel y marcar cada punto donde se cruce una línea de nivel anotando su altura tal como lo muestra el dibujo.

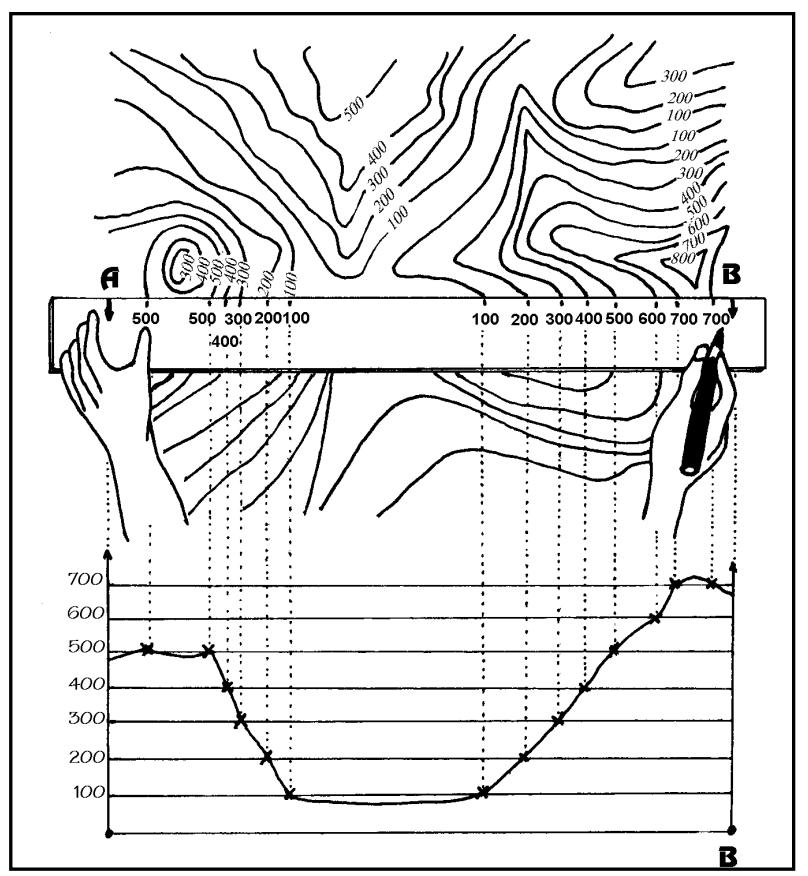


4. En otra hoja, dibuja una línea de la misma longitud que A-B: Esta será la coordenada de distancia.

5. En el punto A, trazar una perpendicular hacia arriba: Esta será la coordenada de altura.

6. Sobre la coordenada de altura trazar líneas de intervalos, cada 5 milímetros. Una para cada altura de nivel: La primera altura, en la coordenada, deberá ser el número más bajo que hayas obtenido al realizar el paso 3.

7. Trasladar las marcas efectuadas en el papel a la coordenada de distancia.

8. Donde se unan las coordenadas de distancia y altura, según las marcas, hacer una cruz.

9. Unir las cruces. La línea resultante te mostrará el perfil transversal de la ruta A-B.

10. Si la coordenada de altura está dibujada también en escala podrás estimar la distancia horizontal real y a partir de esos datos, planificar la marcha.

11. Si el perfil resultante resulta con poco resalte se puede realzar la coordenada de altura ampliando la escala por 5 veces la distancia.

12. El perfil es aconsejable presentarle con formas redondeadas, no "picudas".

Tus resultados servirán como una estimación muy útil fiable, pero no como una medición exacta y precisa.

\section{Paradas y zonas de descanso o de interés:}

Las paradas dependerán de las características del grupo, tanto por la edad, como de la experiencia, entrenamiento y número, ya que si participa mucha gente en una marcha se rompe el ritmo y las paradas se prolongan.

Si el grupo es numeroso y de edades tempranas, conviene realizar una primera parada al poco tiempo de comenzar la marchay asi poder ajustarse las prendas, cordones, mochila, y aprovechar para dar algunos consejos sobre el ritmo, grupos que deben ir delante o atrás etc. Después se suele parar cada hora u hora y media unos cinco o diez minutos, ya que no conviene que las paradas sean largas por la pereza que en muchos casos se experimenta a reanudar la marcha.

Reseña histórica del recorrido, costumbres, fiestas, curiosidades, etc.

Las marchas son uno de los mejores medios que tenemos a nuestro alcance para conocer la historia, el patrimonio cultural, las costumbres, la gastronomía, la tipología edificatoria etc. En todo momento deberemos tener presente, que somos nosotros los que llegamos a un ambiente normalmente distinto del de procedencia, y por ello debemos respetar las formas de vida, los usos y costumbres, evitando las comparaciones y juicios que no vienen al caso la mayoría de las veces. El querer imponer las modas, o «ventajas» propias de las urbes está fuera de lugar, siendo más propio de personas cultas e inteligentes la adaptación e integración allá donde se fuere.

No se debería mencionar siquiera el respeto que debemos adoptar en todo momento con el medio, de jándolo mejor que a nuestra llegada, y educando en todo momento a aquellas personas que estén a nuestro mando. El marchar en fila por prados sin segar, el cerrar las portillas que encontramos cerradas, evitar los chillidos, respetar las propiedades, son «detalles» que deberemos tener presente.

\section{Permisos necesarios:}

Uno de los puntos que muchas veces pasamos por alto hasta el último momento es el referido a los permisos, no nos referimos aquí al de los padres o tutores (necesario), sino a otro también formal y necesario.

Permisos de ferrocarril (bicicletas), billetes con reducción (grupo), visitas a ermitas, museos, estancia en propiedades privadas o públicas, seguros de responsabilidad civil, aviso a los seguros escolares o a las mutuas de seguro en su caso, de acampada, de fuego etc. son algunos de los más comunes, pero además debemos tener en cuenta aquellos más específicos de las zonas por donde nos movamos, como la compatibilidad de horarios para la pesca, los descensos de ríos, las cacerías, etc.

\section{Itinerarios alternativos y zonas de seguridad}

Algunas veces, una expedición, a pesar de su estudio o preparación puede encontrarse con una serie de obstáculos imprevisibles; por esta razón, deben estudiarse itinerarios o actividades alternativas (visitas artísticas, juegos o competiciones deportivas...).

Se hace necesario en este punto tener previsto algunas salidas de emergencia para solucionar imprevistos (accidentes, meteorología adversa, cortes de camino etc.).

\section{Actividades complementarias}

Cuando no es posible realizar la actividad principal, o cuando la estancia se va a prolongar durante algún tiempo, podemos realizar en estos casos multitud de actividades.

Las podemos clasificar en actividades complementarias de utilidad, pedagógicas y recreativas, científicas, deportivas y de supervivencia.

En el cuadro mostrada continuación podemos observar algunas de ellas.

\section{ACTIVIDADES COMPLEMENTARIAS}
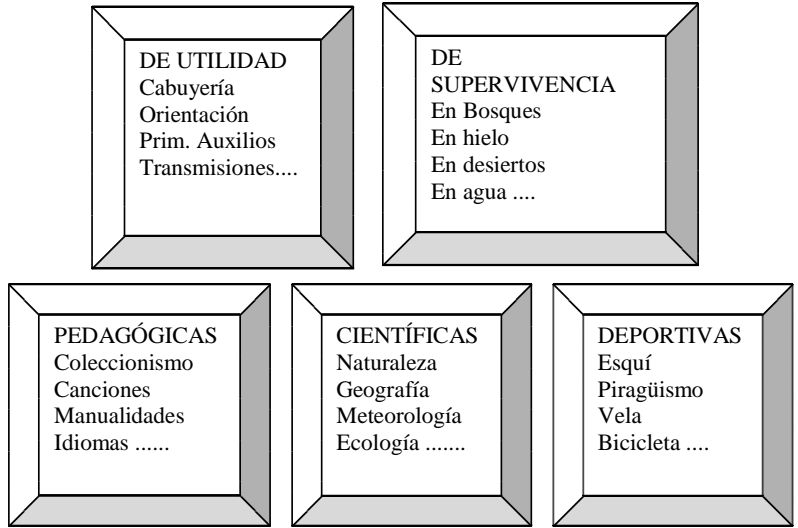

¿Qué es una tarjeta de ruta? (rutómetro) Si quisieras registrar los datos de tu recorrido, antes o durante tu marcha, te conviene preparar una Tarjeta de Ruta. Esta tarjeta te servirá para anotar los sitios por los que pasas y su ubicación en el mapa. Incluye los rumbos, horas, y referencias de cada sitio, altitud, km recorridos, y otros datos de interés. Lógicamente este trabajo se realiza toda vez que quieras revisar los datos del itinerario, luego de haberlo recorrido.

Consejos prácticos para la realización de una marcha

La marcha debe iniciarse lentamente. 


\begin{tabular}{|c|c|c|c|c|c|c|}
\hline \multicolumn{7}{|c|}{$\begin{array}{c}\text { A.D. PEÑA DE LOS JUEVES } \\
\text { I JORNADA DE CONVIVENCIA EN BICICLETA DE MONTAÑA } \\
\text { TRAVESIA DE LA RUTA DEL LAGO CURAVACAS AL MONUMENTO DEL OSO (PJCOS DE } \\
\text { EUROPA) } \\
\end{array}$} \\
\hline \begin{tabular}{|c|} 
Kms. \\
Parciales
\end{tabular} & $\begin{array}{l}\text { Kms. } \\
\text { Totales }\end{array}$ & $\begin{array}{c}\text { Zona } \\
\text { geográfica }\end{array}$ & Cota & $\begin{array}{c}\text { Descripción del } \\
\text { terreno y } \\
\text { orientación }\end{array}$ & Croquis e información & $\begin{array}{l}\text { Tie } \\
\text { mp. }\end{array}$ \\
\hline 0 & 0 & $\begin{array}{l}\text { Potes } \\
\text { Mercado }\end{array}$ & $x$ & $\begin{array}{c}\text { Plaza } \\
\text { asfaltada. } \\
\text { Tomar } \\
\text { carretera } x\end{array}$ & & 0 \\
\hline 0,150 & 0,150 & Cruce I & $x$ & Carretera $n^{\circ}$ & & \\
\hline 0,650 & 0,800 & $\begin{array}{l}\text { Potes } \\
\text { carretera } \\
\quad n^{\circ}\end{array}$ & $x$ & $\begin{array}{l}\text { Carretera } \\
\text { Recto }\end{array}$ & & $1^{\prime}$ \\
\hline 0,900 & 1,700 & $\begin{array}{l}\text { Ojedo } \\
\text { Carretera } \\
n^{\circ}\end{array}$ & $x$ & $\begin{array}{c}\text { Puente de } \\
\text { Ojedo } \\
\text { Cruce D } \\
\text { Dirección } \\
\text { Palencia }\end{array}$ & $\overline{\frac{P a l e n c i a}{0}}$ & $X$ \\
\hline 1,500 & 3,200 & Frama & $x$ & $\begin{array}{c}\text { Carretera De } \\
\text { Frama. Recto. }\end{array}$ & & $x$ \\
\hline 2,400 & 5,600 & $\begin{array}{c}\text { Cabezón } \\
\text { de Liébana }\end{array}$ & $X$ & $\begin{array}{l}\text { Carretera de } \\
\text { Cabezón de } \\
\text { Liébana. Recto }\end{array}$ & & $x$ \\
\hline 1,800 & 7,400 & $\begin{array}{c}\text { Puente } \\
\text { Asnil }\end{array}$ & $x$ & $\begin{array}{c}\text { Carretera de } \\
\text { Puente Asnil no }\end{array}$ & & $x$ \\
\hline 6,400 & 13,8 & & $x$ & $\begin{array}{c}\text { ¿Atención! } \\
\text { Cruce D. } \\
\text { Dejar carretera } \\
\text { general }\end{array}$ & pendes & $x$ \\
\hline & & & & etc. & & \\
\hline
\end{tabular}

- Marchar por la izquierda y en columna en las zonas de tráfico rodado. Si es de noche o con poca visibilidad, llevar luz o elementos reflectantes en la cabeza y en la cola de la columna.

- Evitar la marcha prolongada por camino asfaltado sobre todo durante el verano.

- Los «atajos» se deben coger al bajar, pero no al subir.

- El profesor o el monitor debe ir a la cabeza de la columna, seguido de los más «débiles», al final irán los más resistentes.
- Ni andando, ni durante los descansos se tirará deshechos, salvo que sea alimento o materia orgánica rápidamente degradable.

- Los suaves cambios de ritmo en la cabeza de la columna son violentos en la cola de la misma y repercuten de manera importante.

- La bota estará usada y suavizada antes de una marcha prolongada; las uñas cortas y los pies limpios.

- No se deben atravesar sembrados o prados sin segar; en el caso de tener que hacerlo por necesidad, se ha de marchar por la huella del de delante y a ser posible por la linde. 
- Se habrán de respetar siempre las propiedades privadas. Las puertas se deben dejar cómo se encuentran, abiertas o cerradas. Los animales del ganado deben ser «rodeados», si es posible, sin dar voces ni asustarlos.

- Cada uno controla y ayuda a los que tienen inmediatamente delante o inmediatamente detrás del grupo dando la voz de alarma en caso de accidente o dificultad grave.

- Siempre hay que prestar mucha atención al fuego (cigarrillos, cerillas, cristales...).

- Prever el equipo y el repuesto según la época del año y la actividad a desarrollar.

- Llevar la mochila equilibrada, cargando el peso sobre los hombros, bien sujeta y evitando cantos, picos, etc. en la zona que da a la espalda.

- Siempre se debe llevar un control del grupo, se pasará lista, contará o revisará a sus componentes al comenzar la marcha y al terminarla y también en los descansos.

- Es preferible caminar por sendas o caminos claros, si los hubiere.

- Paradas, las necesarias, pero cortas y en lugares resguardados del viento, la lluvia o el sol fuerte.

- Subir y bajar las pendientes pronunciadas y prolongadas en zig-zag. Son más peligrosas las bajadas que las subidas, por producir «fatigas localizadas», en las rodillas y en los tobillos. El trabajo muscular es de tipo excéntrico (contracciones), su localización muscular en los cuádriceps puede producir agujetas.

- En la primera parada deben ajustarse las botas, macuto, y añadir o quitar la ropa para la etapa siguiente.

- No permitir disgregaciones ni iniciativas individuales durante la actividad.

- Adaptarse a las posibilidades de los más débiles.

- Procurar llevar siempre una cuerda y un cuchillo o navaja grande, o bien de múltiples usos.

- No olvidarse del plano, mapa y la brújula, o GPS.

- El profesor/monitor debe tener en cuenta que no se trata de su propia diversión, sino que debe ser el gran sacrificado.

- En ningún momento se buscará el riesgo.

- Servirse siempre que los haya, de los alumnos o miembros aventajados, y seguir las normas de la pedagogía general sobre líderes del grupo, etc.

- Llevar botiquín con lo más imprescindible.

- Llevar otros elementos sanitarios y de socorro a juicio del profesor.

\section{Glosario}

A continuación, definimos alguno de los términos más usados en las actividades realizadas en aire libre.
ABRIGO: paraje defendido de los vientos. Covacha. Refugio.

ACEBAL: terreno poblado de acebos.

AGUJA: pico montañoso muy delgado y agudo.

ALCOR : Colina, cerro.

ALGAR: cueva o caverna.

APRISCO : refugio para el ganado.

ARGAYO: masa de piedras y tierra que se desprende y cae por la ladera de un monte.

ARGUMA: argoma, escajo.

ARISTA: Reunión de dos vertientes en una montaña.

ASOMO: lugar con vistas al vacío.

ASUBIADERO: caseta o marquesina que sirve para guarecerse de la lluvia.

BEYO: desfiladero, garganta.

BORDA: choza o casilla aislada para guarecer el ganado (Pirineos).

BRAÑA: pasto de montaña, normalmente aprovechado por ganado en los meses de verano.

BREZO: matorral.

CABAÑAL: camino o vía pecuaria por donde pasan las cabañas o ganados. Cobertizo para guardar el ganado.

CABEZA: cumbre con abundantes pastizales, en las inmediaciones de la alta montaña.

CAMBERA: camino carretero.

CANAL: vaguada estrecha, emparedada y de gran inclinación. Concavidad vertical, de gran amplitud y altura.

CANCHAL: pedrera.

CANTO: elevación rocosa y escarpada.

CAÑÓN: profunda garganta que excavan las aguas de los ríos encajándose en el terreno.

CÁRCAVA: Barranco o zanja grande y estrecha que hacen las avenidas del agua.

CEMBA: nevero que perdura todo el verano.

CERRO: elevación del terreno, por lo común peñascosa, aislada y de menor altura que el monte con montaña.

CIRCO: depresión de forma sencilla y paredes verticales o de gran inclinación.

COLLADO: depresión entre dos montañas, por donde es fácil el paso.

CONCHA: sendero en la roca, resbaladizo y convexo.

CORDAL: cuerda imaginaria que une las cumbres de una cordillera o sierra.

CORDILLERA: serie de montañas enlazadas entre sí y cuyo conjunto forma una individualidad geográfica.

CORNISA: saliente rocoso en el borde de una meseta por medio. Ladera de una montaña o cerro por el que se puede pasar, con más o menos peligro.

CORTAFUEGOS: zanja que se deja sin vegetación en los montes para que en caso de incendio el fuego no la atraviese. 
COTERO: pequeña elevación.

CUENYE: paso difícil. Zona baja y con paso en un terreno accidentado.

CUETO: final aislado y de fácil acceso. Vertientes regulares y cima cónica o redondeada.

DOLINA: depresión cárstica producida por la disolución de la caliza o bien por el hundimiento del terreno por la circulación subterránea de aguas.

ESCAJO: argoma, aulaga, tojo.

ESCOBIO: angostura, hoz, garganta o paso estrecho en una montaña o en un río.

FRESNEDA: paraje cubierto de fresnos.

FREYTA: quebrada abierta por un torrente en una ladera.

FURNIA: caverna.

GARGANTA: cauce estrecho y profundo abierto por un río, cuyas paredes son casi verticales.

GARMA: lapiaz, pedrera.

GARRIGA: maleza, matorral propio de ambientes mediterráneos.

GAVIA: zanja de desagüe o de límite de propiedades.

HELGUERA: helechar. Zona cubierta de helechos.

HERBAZAL: sitio poblado de hierbas.

HITO: mojón o poste para marcar linderos de términos municipales, heredades, etc... Pequeño montón de piedras para señalar un camino (jito).

HORADADA: agujero que atraviesa de parte a parte. Túnel.

HORCADO/A/INA: paso divisorio transitable entre ladera en pendientes muy fuertes.

HOYO/JOU: concavidad u hondura formada natural o artificialmente en la tierra.

HOZ: angostura de un valle profundo o curva que forma un río encajándose cuyas figuras recuerdan las del instrumento que le da nombre.

IBÓN: laguna situada a la altura y alimentada por la fusión de las nieves. Lámina de agua, resto de antiguo glaciar.

INVERNAL: Cabañal donde se recoge y alimenta al ganado en los meses de invierno.

KARST/carst: conjunto de formaciones superficiales o subterráneas producidas en las rocas calizas por la circulación de agua cargada de carbónico.

LAJA: placa de roca.

LAPIAZ: garma. Formación cárstica superficial, de tamaño variable y sumamente irregular.

LOMA/lomba: altura pequeña y prolongada del terreno; puede ser protuberancia aislada. Formar una serie de alineaciones o su prolongación.

LLAMBRÍA: superficie rocosa lisa e inclinada. Parte de un peñasco que forma un plano muy inclinado y pulimentado por la erosión. Llambrión, llambría grande.
LLERA: pedrera.

MAJADA: lugar donde se recoge de noche el ganado y se albergan los pastores.

MEDIAJO/MIDIAJO: lugar elevado donde suele correr el aire, al que acude el ganado en verano para liberarse del calor y los insectos.

MESA: terreno elevado y llano de gran extensión rodeado de valles o barrancos. Cima plana de una montaña.

MESTAS: confluencia de ríos.

MOJÓN: pequeña columna de cemento colocada por el instituto geográfico nacional para señalizar un vértice geodésico, generalmente ubicado en una cumbre.

MORRA: cima llana con dos cortes verticales por ambos lados de la crestería. Saliente rocoso rodeado.

MORRENA: conjunto de rocas acarreadas y depositadas por un glaciar.

NEVERO: acumulación de nieve persistente.

OTERO: cerro aislado que domina un llano.

PANDO: terreno casi llano situado entre dos montañas.

PEÑALAR: ladera cubierta por canto suelto de distinto tamaño y difícil tránsito.

PINDIO: empinado.

RASA: llanura alta, despejada con paso.

REPECHO: pendiente pronunciada pero breve.

\section{RIEGA: regato.}

RIMAYA: grieta formada entre una masa de nieve y la roca.

SEDO: paso accidentado a través de la roca desnuda

SENDA: camino angosto más estrecho que la vereda, en el que sólo cabe una persona o caballería.

SURGENCIA: lugar por donde surge o brota agua.

TENADA: cabaña o caseta de grandes dimensiones destinada a albergar ganado en las zonas altas.

TORCA: depresión circular del suelo, con bordes escarpados en forma de embudo, en la que se suman las aguas superficiales.

TORNO: revuelta. Zig-zag de un camino o senda.

UMBRÍA: vertiente menos iluminada por el sol, opuesta a la solana. En el hemisferio norte se corresponde con las laderas orientadas al norte.

VAGUADA: zona deprimida del terreno por la que discurren las aguas de escorrentía sin formar necesariamente río, arroyo ni torrente.

VALLE: terreno más o menos llano o cóncavo rodeado por montañas.

VEGA: zona próxima a un río de suelo llano y fértil.

VENERO: manantial de agua.

VENTISQUERO: lugar donde naturalmente se acumula y conserva la nieve.

VISO: lugar alto desde donde es visible mucho terreno. 
VIVAC: sitio arreglado, acondicionado para pasar la noche en el monte cuando no se dispone de tienda o refugio (vivaquear).

\section{Referencias Bibliográficas}

Ascaso Martorell et al. Actividades en la naturaleza. MEC.1996.

Bello Collado L. M. Aire libre. OJE.

Cendrero Ucieda y otros. Guía de la naturaleza de Cantrabria.E. Estudio 1993

Chevalier y LECHARTIER. El libro de los primeros socorros. E. Grims 1973.

Costa Pablo J. Cánovas / Lázaro Giménez Martínez. Orientación en la naturaleza http:// www.paseosenchile.cl/espanol/tecdecamp/naturale.htm.

Corberó M .V. Y OTROS. Trabajar mapas. Alhambra 1988.

Díaz De Terán, Flor, Francés y otros. ANIDA itinerarios 1 y 2 A.G. Resma. Santander 1984

D.N.E.F.D. Guía didáctica del Área de Expresión dinámica.1974.
García Moro R.,Orellana Mallavia J.:Diseño y organización de itinerarios y marchas a pie. Curso albergue juvenil de Arbejal. 1994.

García B. G. Cimiano y G. Campo. Cuaderno de orientación. CEP 1991.

http://www.desnivel.es/tus_paginas/orientacion/ object.php?o=3148

http://www.siemprescout.org/index.html.

$h+t p: / / w w w . p a s e o s e n c h i l e . c l / e s p a n o l / t e c d e c a m p /$ naturale. htm

$h+t p: / / w w w . d e s n i v e l . e s / t u s \_p a g i n a s / o r i e n t a c i o n /$ object.php?o=3148.

http://www.elcorreodigital.com/guiaocio/aunpaso/ aunpaso080599.html\#datos.25/02/05

Lourenço Juan Manuel. Cuaderno de campo No $8 \mathrm{http}: /$ /www. geocities.com/ jmlweb2000/jmlweb.htm.

Querol Carceller $S$ et alt; Créditos variables de actividades en la naturaleza. Paidotribo

Sáiz De Omeñaca, Antón, Calatayud y otros. Utilización didáctica del medio ambiente. ICE Universidad de Cantabria.

Sañudo José Antonio. Rutómetro al lago Curavacas en todo terreno. Inédito. 1995.

- La actividad se encuentra homologada por la Consejería de Educación y Ciencia ya que se encuentra actualmente Subvencionada por dicha Consejería.

- Se ha solicitado la correspondiente convalidación por los créditos de libre elección en la Universidad de Cádiz.

\section{INFORMACIÓN}

La lista de admitidos, así como el programa definitivo podrá consultarse en la página Web:

\section{www.csi-csif.es $\backslash$ andalucialcadiz}

La organización dispone de un número de plazas limitadas para los asistentes en alojamiento de estudiantes en habitación doble desde el viernes tarde hasta el domingo al mediodía.

Precio por noche : 15 .

Antes de abonar consultar disponibilidad en el numero de teléfono: $\mathbf{6 6 0 7 2 9 7 5 3}$

Puede solicitar información de las Jornadas en el siguiente teléfono: $\mathbf{6 6 0 7 2 9 7 5 3}$ 